\title{
Perceptual learning can reverse subliminal priming effects
}

\author{
Anna Przekoracka-Krawczyk \\ Adam Mickiewicz University, Poznań, Poland \\ AND \\ PIOTR JAŚKOWSKI \\ University of Finance and Management, Warsaw, Poland
}

\begin{abstract}
Masked primes presented prior to a target can result in inverse priming (i.e., benefits on trials in which the prime and the target are mapped onto opposite responses). In five experiments, time-of-task effects on subliminal priming of motor responses were investigated. First, we replicated Klapp and Hinkley's (2002) finding that the priming effect is initially straight (i.e., it benefits congruent trials, in which the prime and targets are mapped onto the same response) or absent, and only later reverses (i.e., faster responses in incongruent than in congruent trials). We show that the presentation of the mask plays a crucial role in this reversal and that the reversal occurs later if the mask pattern is very complex. We suggest that perceptual learning improves the recognition of taskrelevant features. Once recognized, these features can trigger the preparation of the alternative response and/or inhibit the prime-activated response. These findings support an active role of the mask in priming.
\end{abstract}

Recent results have provided strong evidence that unconscious stimuli can affect people's actions (Ansorge, Klotz, \& Neumann, 1998; Di Lollo, Bischof, \& Dixon, 1993; Jaśkowski, Skalska, \& Verleger, 2003). In such experiments, participants typically make speeded choice responses to two stimuli-for instance, arrows pointing to the left or the right. These figures are preceded, however, by briefly displayed stimuli (primes) that are similar or identical to the target stimuli. Usually, a neutral mask is inserted between the prime and target stimuli in order to make the prime invisible. Even though the primes are not identifiable, responses to the target stimuli nonetheless are faster and more accurate when the prime and target both call for the same response (a congruent trial) than when the stimuli call for different responses (an incongruent trial).

These findings can be accounted for by the assumption that the masked prime is automatically processed, so that the response is prepared according to both the participant's intentions (or the experimenter's instructions; e.g., "respond with the left/right hand to a left-/right-pointing arrow") and the prime identity. Thus, preparation is already in progress when the target stimulus appears, leading to benefits in congruent trials and costs in incongruent trials.

However, subliminal priming does not always produce benefits for congruent trials and costs for incongruent trials (straight priming). Eimer and Schlaghecken (1998) showed that under some conditions, people perform better for incongruent than for congruent trials (inverse priming). In their experiments, they used double arrows pointing to the left or right as the prime and target stimuli. Left- and right-pointing double arrows superimposed upon one another formed the mask. In this case, responses were longer and less accurate when prime and target were congruent than when they were incongruent. To account for this inverse priming, Eimer and Schlaghecken later formulated the self-inhibition (SI) hypothesis (Eimer, Schubö, \& Schlaghecken, 2002; Schlaghecken \& Eimer, 2002), which suggested that a self-inhibition phase follows prime-evoked activation. When a target appears shortly after a prime, the response preparation triggered by the target falls during the activation phase, leading to straight priming. For longer prime-target intervals, however, the inhibitory process predominates, leading to inverse priming. This theory accounts for Schlaghecken and Eimer's earlier findings (Schlaghecken \& Eimer, 1997, $2000,2001)$ that inverse priming occurs only if the delay between prime and target is sufficiently long.

However, some authors have recently suggested (Jaśkowski \& Przekoracka-Krawczyk, 2005; Lleras \& Enns, 2004; Verleger, Jaśkowski, Aydemir, van der Lubbe, \& Groen, 2004) that the mask itself triggers the inhibitory processes that eventually lead to inverse priming. Indeed, in the majority of Eimer and Schlaghecken's experiments (Eimer, 1999; Eimer \& Schlaghecken, 2001; Eimer et al., 2002; Schlaghecken \& Eimer, 1997, 2000, 2001), the mask was composed of two double arrows pointing

A. Przekoracka-Krawczyk, aniaprzek@poczta.onet.pl 
in opposite directions. Such a mask added the opposite target stimulus to the briefly presented prime; therefore, the mask might have activated the response opposite to the prime, which might have led to delayed responses in congruent trials and speeded responses in incongruent trials. This observation led Lleras and Enns (2004) and Verleger et al. (2004) to formulate similar hypotheses that attributed inverse priming to the fact that the mask contained arrow-like task-relevant elements, and therefore acted as a second prime. In Lleras and Enns's object updating (OU) hypothesis, information conveyed by each stimulus is automatically analyzed for relevant features. Features found in the prime form a scene representation and evoke initial response preparation. Features presented in the mask in their turn update the scene representation and change response preparation accordingly. If the mask is formed from two primes calling for different responses, the update provides the features of the arrow opposite to that in the prime, and the preparation of the opposite response is started. This hypothesis was corroborated by Lleras and Enns as well as by Verleger et al. (2004), who showed that straight priming occurred when the mask had no relevant features. In Verleger et al.'s (2004) active mask (AM) hypothesis, they also emphasized the arrow-like character of the mask as a contributor to inverse priming. According to this hypothesis, arrows are rather special stimuli that immediately induce a tendency to shift attention (Verleger, Ewers, \& Jaśkowski, 2006; Verleger, Vollmer, Wauschkuhn, van der Lubbe, \& Wascher, 2000) and that modify the preparatory state (Praamstra, Boutsen, \& Humphreys, 2005); a sequence of arrow primes and arrow masks thus induces a "flip-flop" of tendencies, first to one side, then to the other (Jaśkowski \& Ślósarek, in press; Verleger, Görgen, \& Jaśkowski, 2005).

The present study was motivated by a finding of Klapp and Hinkley (2002). Interestingly, they did not observe stable inverse priming immediately, but only on the second and third experimental day, suggesting that some kind of perceptual or motoric learning is involved in determining the priming effect. They provided no convincing explanations of this initial period of instability. One of their suggestions was that inverse priming increases with time because of a decrease in the ability to detect primes, but even they considered this scenario to be rather implausible. Another possibility was that inverse priming is caused by the prime-mask interaction and occurs only when participants are able to recognize searched-for features in the mask. If the features are easy to find (as in the case of a mask formed by two oppositely pointing arrows), inverse priming appears at the very beginning of a session. If, however, the mask structure is more complex, participants need more time to extract the relevant features from it. This account can easily be incorporated into the OU hypothesis, but it is difficult to reconcile with the SI hypothesis.

In the following pages, we report on five experiments designed to address these predictions more directly. First, we successfully replicated Klapp and Hinkley's (2002) finding: The priming effect was initially straight or absent, and only later became inverse (Experiment 1). We then showed that this reversal required the presence of the mask during the initial training phase (Experiment 2), but not of the prime (Experiment 3 ). Then we showed that reversal of the priming effect depended in part on the complexity of the mask (Experiment 4) and that complex masks can generate inverse priming effects for longer intervals (Experiment 5).

\section{GENERAL METHOD}

\section{Participants}

We recruited participants from the student population of Adam Mickiewicz University. All except 2 were right-handed, and all had normal or corrected-to-normal visual acuity.

\section{Apparatus and Stimuli}

The stimuli were displayed on a 17 -in. computer monitor with a refresh rate of $75 \mathrm{~Hz}$ by means of Presentation software (Version 0.55, Neurobehavioral Systems).

Right- and left-pointing double arrows similar to those used by Eimer and Schlaghecken (1998) were used as the primes and targets. The mask was composed of arrows identical to those forming the primes and targets, distributed randomly over a rectangle $1.62^{\circ} \times$ $1.15^{\circ}$. The primes and targets each fit into a rectangle $1.05^{\circ} \times 0.80^{\circ}$ (see Figure 1).

\section{Procedure}

Participants sat in a comfortable armchair in a darkened room in front of the computer monitor. The observation distance was $100 \mathrm{~cm}$.

The experimental session consisted of two parts: a training stage (TRS) followed by a test stage (TSS). Each stage started with a target identification (TI) block during which choice reaction times (RTs) to the target stimuli were measured. Participants had to respond according to the target identity as quickly and accurately as possible by pressing the left or right control key of a standard computer keyboard for a left or a right target arrow, respectively. This block was followed by a prime discrimination block (PD). During the PD block, prime recognition was tested, and no target was displayed. Participants had to guess the prime identity by pressing the left or right control key for a left- or right-pointing priming arrow, without pressure with respect to speed. No visual feedback was provided. In Experiments 2, 3, and 5, the PD blocks were omitted in the training stage.

All experiments were preceded by practice blocks (20 trials each) to ensure that participants understood the instructions.

A trial started with fixation assistance: Four points appeared $5^{\circ}$ above, below, to the left, and to the right of the screen center, moved inward for $0.9 \mathrm{sec}$, and then "crystallized" as a fixation cross. The prime appeared for $13 \mathrm{msec}$ and was replaced with a mask $(107 \mathrm{msec})$; in the TI blocks only, the mask was followed by a tar-

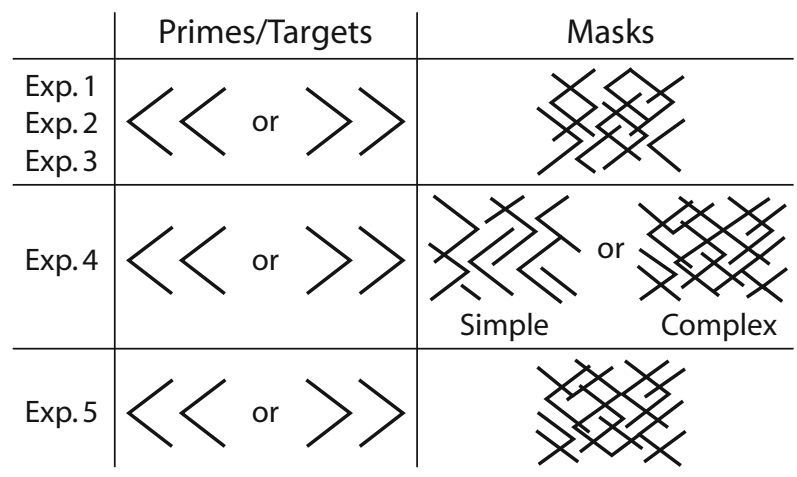

Figure 1. Stimuli used in all experiments. 
get $(107 \mathrm{msec})$. The next trial began $0.8 \mathrm{sec}$ after the participant's response.

\section{Data Analysis}

Mean reaction times (RTs), error rates, and $d$ 's were evaluated statistically by repeated measures ANOVAs.

Moreover, Student's $t$ tests were used to check whether $d^{\prime}$ differed from zero.

\section{EXPERIMENT 1}

In Experiment 1, we sought to replicate Klapp and Hinkley's (2002) main finding concerning the instability of inverse priming. Two sets of TI blocks (TRS and TSS) were performed, with a short pause between them. We expected to find straight priming in the training stage and inverse priming in the test stage.

\section{Method}

Participants. Eleven students (9 females and 2 males, ages 19 30) participated in the experiment.

Procedure. Each TI block consisted of 150 trials (75 congruent, with the arrows in the prime and target pointing to the same side, and 75 incongruent, with the arrows in the prime and target pointing to opposite sides). A PD block of 80 trials was run after each TI block.

\section{Results}

Prime discrimination. After the training stage, $d^{\prime}$ was -0.04 (range, -0.39 to +0.35 ), which corresponds to $48 \%$ correct guesses. Similarly, in the test stage, $d^{\prime}$ was -0.06 (range, -0.29 to +0.11 ), which also corresponds to $48 \%$ correct responses. Student's $t$ tests showed that the $d$ 's were not significantly greater than zero $[t(10)=$ $-0.66, p=.520$, and $t(10)=-1.46, p=.160$, for the TRS and TSS, respectively; see the left bars in Figure 2]. $d$ 's did not differ significantly between the tests $[F(1,10)=$ $0.07, p=.791]$.

RTs and error rates. These results are depicted in Figure 3. There was no reliable difference between mean RTs in the two TI blocks [ 427 and $430 \mathrm{msec}$ for the TRS and TSS; $F(1,10)=0.2, p=.629]$. However, the congruency $\times$ stage interaction was significant $[F(1,10)=$ $8.22, p=.017]$. Contrast analysis revealed that in the TRS there was no reliable effect, as measured by the difference RT(incong) - RT(cong) [+7 msec; $F(1,10)=1.45, p=$ $.257]$, but in the TSS the effect became inverse [ $-14 \mathrm{msec}$; $F(1,10)=6.5, p=.029]$.

Mean error rates were almost identical in both TI blocks $[9 \%$ vs. $8 \%$ for the TRS and TSS; $F(1,10)=0.19, p=$ .67]. The mean priming effect was straight in the TRS [7\% vs. $11 \%$ for congruent vs. incongruent trials; $F(1,10)=$ $5.40, p=.043$ ], and no priming effect occurred in the TSS $[7 \%$ vs. $10 \% ; F(1,10)=3.02, p=.113]$. There were no other significant effects or interactions.

\section{Discussion}

Klapp and Hinkley (2002) found that significant inverse priming occurred only after some practice. The results of Experiment 1 replicated their finding: Inverse priming occurred only in the test stage $(-14 \mathrm{msec})$, and in the training stage no effect was found at all $(+7 \mathrm{msec})$.

The results of our prime identification ability test did not, however, support Klapp and Hinkley's (2002) suggestion that the effect stems from a paradoxical decrease in prime discrimination ability due to training. Unlike Klapp and Hinkley, we found no changes in masking efficiency with training (the $d^{\prime}$ s did not differ reliably from zero in the two PD blocks).

We claim that the reversal of the priming effect was due to perceptual learning of the mask rather than to a decrease in prime visibility. According to our hypothesis, learning may allow the visual system to recognize arrows more efficiently among the lines forming the mask. The mask was composed of arrows strewn disconnectedly over an area, making recognition of these elements and their meanings for the task quite difficult. If mask content can modulate the priming effect, then better recognition of mask content should change the effect. Therefore, when the participants' task is to respond to an object, perceptual learning could lead to better recognition of this object in the complex pattern of the mask and, in turn, to a greater interaction between the prime and mask.

However, before we can draw this conclusion, we need to consider some alternative explanations of the effect.
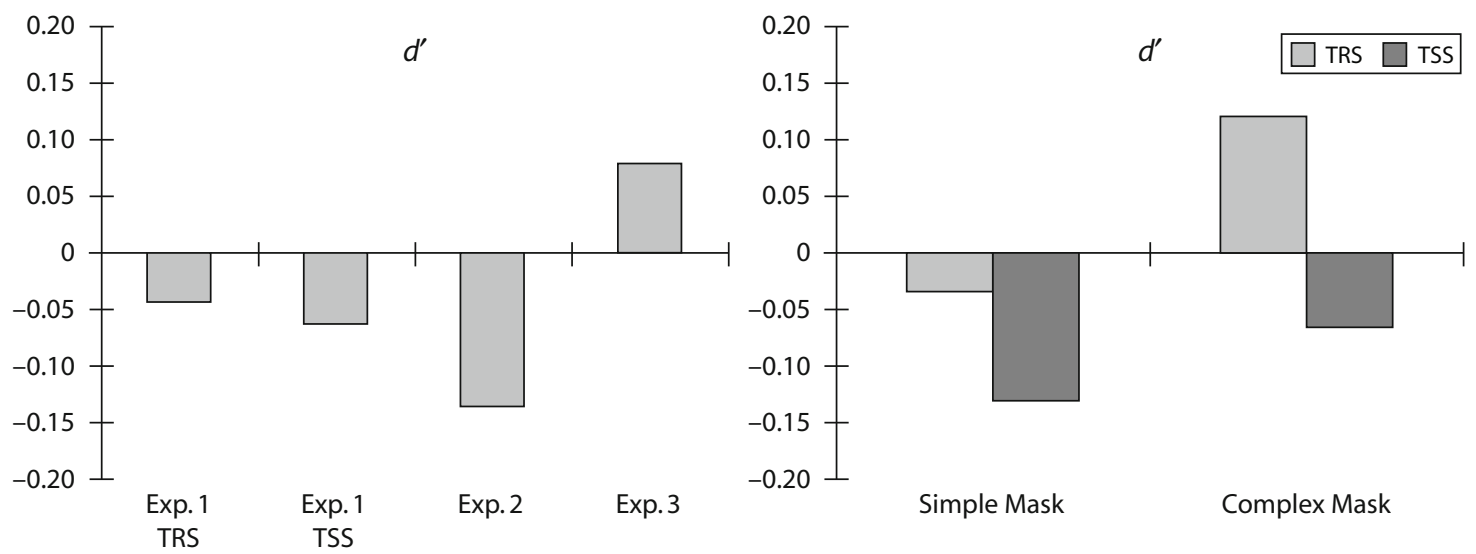

Figure 2. (Left) $d^{\prime}$ measured in Experiments 1, 2, and 3. (Right) $d^{\prime}$ measured in Experiment 4. TRS, training stage; TSS, test stage. 

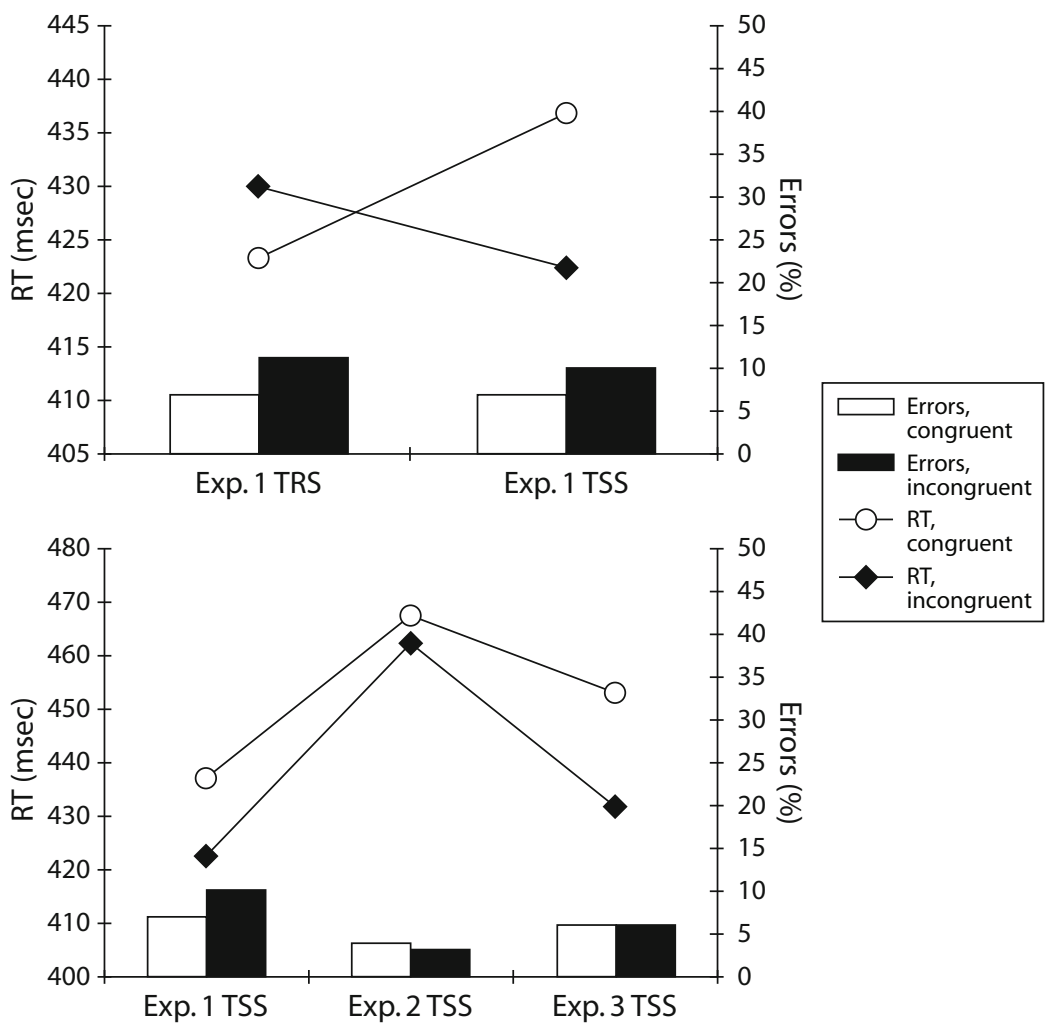

Figure 3. (Top) Mean reaction times (RTs) and error rates of Experiment 1 for the two stages (TRS, training stage; TSS, test stage). (Bottom) Mean RTs and error rates of Experiments 1, 2, and 3 in the TSS. For RTs, congruent trials are denoted by white circles and incongruent trials by black diamonds. For errors, white bars are results for congruent trials and black for incongruent trials.

\section{EXPERIMENT 2}

Experiment 2 was performed in order to examine the possibility that the priming reversal in Experiment 1 was based on some form of motor learning (e.g., an improvement of response choice automaticity) rather than on perceptual learning of relevant features in the mask. To examine this possibility, we removed the prime and mask from the training stage. Therefore, participants now made as many responses to targets as in Experiment 1, but were never exposed to the primes and masks before the end of the TRS.

\section{Method}

Participants. Eleven students ( 7 females and 4 males, ages 1930) participated in the experiment

Procedure. Experiment 2 was identical to Experiment 1 in all respects, except for two modifications: First, primes and masks were both omitted in the TRS, though participants were required to respond to the target arrows as before. In addition, a PD block was run only after the TSS.

\section{Results}

Prime discrimination. The mean $d^{\prime}$ was -0.13 (range, -0.40 to $0.04 ; 47 \%$ correct responses). A Student's $t$ test showed that this value of $d^{\prime}$ was significantly lower than zero $[t(10)=-3.03, p=.007]$.
RTs and error rates. Only data from the TSS were analyzed, and no effect of prime congruency was found. Responses were $5 \mathrm{msec}$ slower in congruent than in incongruent trials [467 vs. $462 \mathrm{msec}$, respectively; $F(1,10)=$ $0.19, p=.672]$ and were equally accurate in both conditions $[4 \%$ vs. $3 \% ; F(1,10)=0.54, p=.479]$.

\section{Discussion}

No priming effect was found in the TSS when the mask and prime were eliminated from the TRS, just as no priming effect was found in the TRS of Experiment 1.

Because the numbers of responses were identical in Experiments 1 and 2 , this result excludes the possibility that motor training is responsible for the reversal of the effect. The mask seems to play an important role. We explored this idea further in Experiment 3.

Unlike in Experiment 1, $d^{\prime}$ in Experiment 2 significantly differed from zero after the TSS. A lower-than-zero $d^{\prime}$ is a rather mysterious result, given that $d^{\prime}$ refers to signal detection sensitivity. However, such results have occasionally been reported (see Erdelyi, 2004; Jaśkowski, 2006). Jaśkowski (2006) previously argued that negative $d^{\prime}$ values mean that prime-mask combinations are distinguishable on a fraction of trials, yet participants are nevertheless unable to recognize what was actually presented before the mask. Rather, they are somehow fooled by such 
prime-mask combinations. Whatever the reason for this outcome, it cannot be used to explain the lack of a priming effect in the present experiment, because if a direct link existed between prime identification and the priming effect, one would expect an even more negative priming effect with a negative $d^{\prime}$ than with $d^{\prime}=0$.

\section{EXPERIMENT 3}

If the mask is responsible for the reversal we discuss, the priming effect should be inverted in the TSS, even when no prime is presented in the TRS. This hypothesis was tested in Experiment 3.

\section{Method}

Participants. Eleven students ( 7 females and 4 males, ages 1930) participated in the experiment.

Procedure. Experiment 3 was identical to Experiment 2, with one exception: In the TRS, the mask was displayed before the target, but no prime.

\section{Results}

Prime discrimination. The mean $d^{\prime}$ was 0.08 (range, -0.34 to $+0.54 ; 52 \%$ correct responses). A Student's $t$ test showed that $d^{\prime}$ did not differ significantly from zero $[t(10)=0.99, p=.333]$.

RTs and error rates. Mean RTs are shown in Figure 3. As expected, responses were slower in congruent than in incongruent trials [453 vs. $432 \mathrm{msec} ; F(1,10)=12.46$, $p=.005]$. Thus, the inverse effect obtained amounted to $-21 \mathrm{msec}$.

The error rate, on the other hand, was the same for congruent and incongruent trials $[6 \%$ for both; $F(1,10)=$ $0.05, p=.831]$.

\section{Discussion}

The inclusion of the mask in the TRS resulted in inverse priming.

When we compare the results obtained in the TSSs across all three experiments, we can see that notable inverse priming appeared only when a mask was presented in the TRS. ${ }^{1}$ This finding suggests that the mask plays a crucial role in reversing the priming effect.

\section{EXPERIMENT 4}

We suggested that multiple presentations of the mask improve participants' ability to extract relevant features from it. This efficient searching leads to an interaction between the features found in the prime, the mask, and the target, eventually culminating in inverse priming.

In Experiment 4, we further tested this hypothesis by using masks of different complexity. If a mask is composed of clearly recognizable arrows, observers are able to extract these elements efficiently, so that inverse priming should occur from the very beginning. If, however, the mask is complex, we assume that extracting the relevant elements is less efficient and the prime--mask interaction weaker, leading to smaller or even to no inverse priming effect. Therefore, as before, the participants in this experiment performed the re- action task in two parts (a training and a test stage) in which both simple and complex masks were displayed blockwise, with the simple mask always in the first half of the test. We expected that inverse priming should (1) be generally smaller for complex than for simple masks and (2) become increasingly negative with training. Moreover, we expected a decrease in inverse priming whenever the simple mask was replaced with the complex mask.

\section{Method}

Participants. Thirteen students ( 6 females and 7 males, ages 19-30) participated in the experiment.

Stimuli and Procedure. As in Experiment 1, the session was divided into two parts. Each part consisted of target identification (TRS in Part 1 and TSS in Part 2) and a prime discrimination block.

Two different types of mask were used. These were composed of arrows pointing randomly to the left or to the right (Figure 1). In the simple mask, arrows did not overlap each other, and participants could recognize them very easily. In the complex mask, lines from the arrows crossed, making extraction of the single arrows more difficult. In addition, three different instances of the complex mask were used in order to render recognition more difficult.

The TRS (196 trials) was subdivided into two halves, with no break between: In the first 98 trials, only the simple mask was presented; in the second, the complex mask was presented instead. After a break, the TSS started, which was identical to the TRS. After each test, the prime identification block was run.

\section{Results}

Prime discrimination. $d^{\prime}$ values (Figure 2) were independent of mask type [ -0.08 vs. 0.03 for the simple and complex masks, respectively; $F(1,12)=3.96, p=.070]$ and stage $[0.04$ vs. -0.10 for the TRS and TSS, respectively; $F(1,12)=4.70, p=.051]$. The interaction between these two variables was not significant $[F(1,12)=0.89$, $p=.365]$.

The mean $d^{\prime}$ in the TRS was -0.035 for the simple mask (range, -0.55 to $0.46 ; 50 \%$ correct responses) and 0.12 for the complex mask (range, -0.26 to $0.62 ; 51 \%$ correct responses). Student's $t$ tests showed that both of these $d^{\prime}$ s did not differ reliably from zero $[t(12)=-0.53$, $p=.599 ; t(12)=1.45, p=.161$, for the simple and complex masks, respectively].

Similarly, primes were not visible in the TSS. $d^{\prime}$ for the simple masks was -0.13 [range, -0.55 to $0.09 ; 48 \%$ correct responses; $t(12)=-1.95, p=.062]$ and -0.07 for the complex mask [range, -0.24 to $0.24 ; 49 \%$ correct responses; $t(12)=-1.59, p=.125]$.

RTs and error rates. RTs (see Figure 4) were also independent of type of mask [429 vs. $436 \mathrm{msec}$ for the simple and complex masks, respectively; $F(1,12)=1.50$, $p=.244$ ] and experimental stage [436 vs. $429 \mathrm{msec}$ for the TRS and TSS, respectively; $F(1,12)=0.48, p=.503]$. The mean priming effect was numerically inverse, but not statistically significant [RT(incong) - RT(cong) = $-10 \mathrm{msec} ; F(1,12)=2.55, p=.136]$. However, the congruency $\times$ stage interaction was significant $[F(1,12)=$ $7.88, p=.016]$. Contrast analysis revealed no reliable priming effect in the TRS [437 vs. $435 \mathrm{msec}$ for congruent and incongruent trials, respectively; $F(1,12)=0.118, p=$ .737] and inverse priming in the TSS [438 vs. $421 \mathrm{msec}$; $F(1,12)=8.22, p=.014]$. 


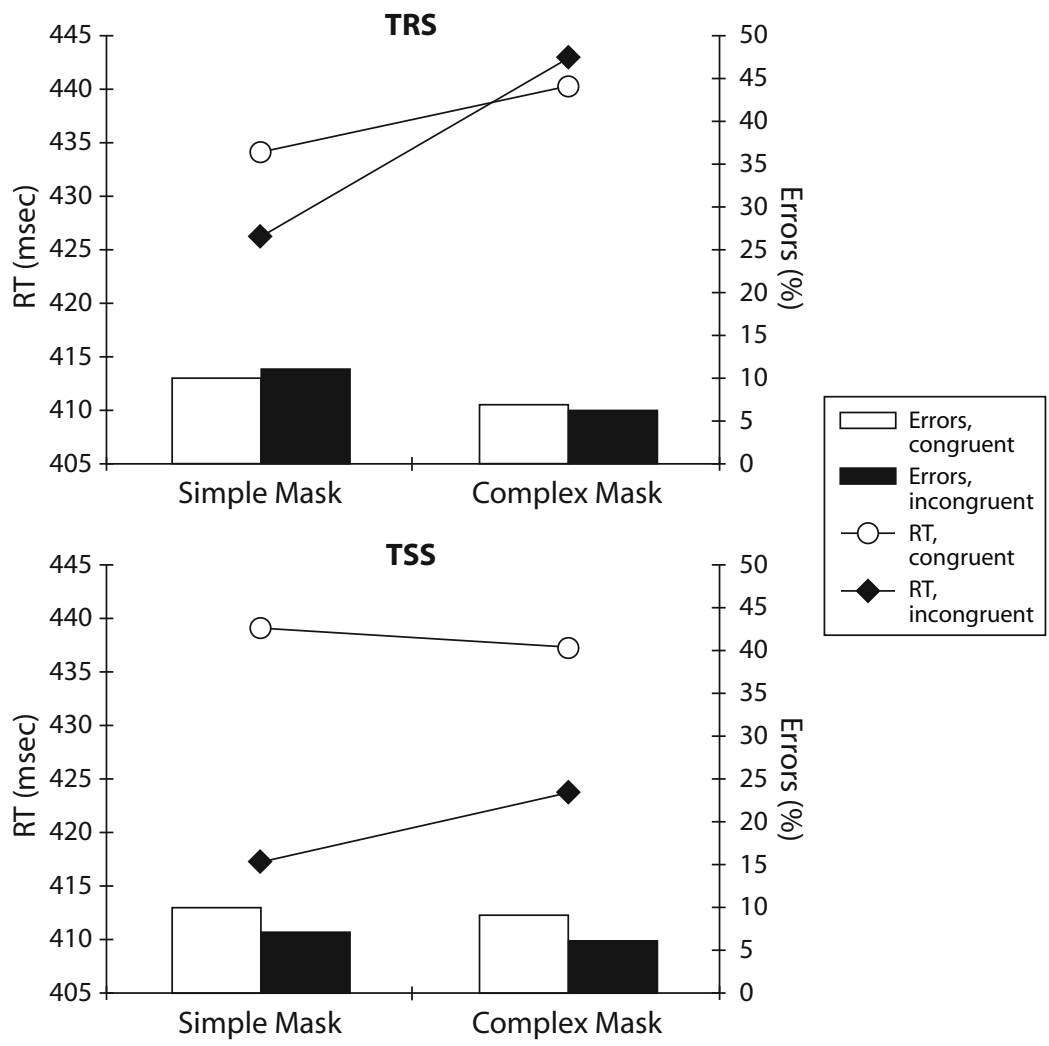

Figure 4. Mean reaction times (RTs) and error rates from Experiment 4, as a function of mask complexity. (Top) Training stage. (Bottom) Test stage. For RTs, congruent trials are denoted by white circles and incongruent trials by black diamonds. For errors, white bars are results for congruent trials and black for incongruent trials.

We also found a significant congruency $\times$ type of mask interaction $[F(1,12)=6.34, p=.027]$. As revealed by contrast analysis, inverse priming occurred for the simple mask [437 vs. $422 \mathrm{msec}$ for congruent and incongruent trials, respectively; $F(1,12)=4.87, p=.040]$, but there was no effect for the complex mask [439 vs. $433 \mathrm{msec}$; $F(1,12)=0.68, p=.425]$.

The three-way interaction (stage $\times$ type of mask $\times$ congruency) was not significant $[F(1,12)=0.06, p=.816]$.

Only the type of mask affected the error rate. Participants made more incorrect responses with the simple than with the complex mask $[10 \%$ vs. $7 \% ; F(1,12)=10.67$, $p=.007]$. Neither stage $[F(1,12)=0.03, p=.865]$ nor congruency $[F(1,12)=2.60, p=.133]$ was found to be significant, and no interaction was found.

\section{Discussion}

As expected, the priming effect was more negative for the simple mask than for the complex one. Moreover, the priming effect became less negative whenever trials with the simple mask were replaced by trials with a complex mask. This corroborates our hypothesis that after an abrupt change from a simple to a complex mask, the relevant elements in the mask became unrecognizable, preventing their interaction with the prime and target elements; therefore, the action initiated by the prime continued. Training improved participants' ability to recognize the relevant features in the mask, rendering the priming effect more negative for both masks in the TSS than in the TRS.

One could argue that these results do not fully corroborate our hypothesis, since the three-way interaction failed to reach significance, and one can expect the learning effect to be faster with simple than with complex masks. Arguably, however, the lack of such an interaction may have resulted from a ceiling effect that occurred with the simple mask: After some practice, the relevant elements were recognizable in the mask, and further practice thus could not increase the priming effect any more.

Mean $d^{\prime}$ s did not differ from zero in the separate conditions. However, an almost significant increase in $d^{\prime}$ with practice was noted (i.e., $d^{\prime}$ was slightly more positive in the TSS than in the TRS). One might wonder whether this increase could be causally responsible for the decrease in the priming effect. It should be noted, however, that this result may not be taken as an argument in favor of Klapp and Hinkley's (2002) suggestion that practicing leads to a decrease in $d^{\prime}$, which in turn implies a more negative priming effect.

\section{EXPERIMENT 5}

In Experiment 5, we tested one more prediction of our hypothesis. The recognition of relevant features in a mask is a time-consuming process, and therefore the mask content can influence the preparation of the motor response 
only after some time from the mask onset. Therefore, we expected that even for a complex mask, inverse priming should occur for longer mask-target intervals (ISIs).

\section{Method}

Participants. Twelve students ( 5 females and 7 males, ages 19 28) participated in the experiment.

Stimuli and Procedure. Experiment 5 was virtually identical to Experiment 4, with the exception of four changes: (1) We used only complex masks; (2) the target was presented 0,53 , or $80 \mathrm{msec}$ after mask offset (ISI 0 , ISI 53, or ISI 80 conditions), in random order; (3) we executed only one prime discrimination block, after the TSS; (4) in the prime discrimination block, three prime durations were applied $(13,53$, or $80 \mathrm{msec})$ in order to motivate participants in recognizing the primes. ${ }^{2}$

\section{Results}

Prime discrimination. Despite the fact that we used, in our view, a more sensitive prime discrimination procedure (three prime durations), $d^{\prime}$ for the shortest prime (13 msec) was 0.19 (range, -0.30 to 0.70 ), which corresponds to $55 \%$ correct responses. A Student's $t$ test showed that $d^{\prime}$ was not significantly higher than zero $[t(12)=1.94, p=.067]$, which suggests that primes were not consciously perceived.

RTs. RTs (see Figure 5) were not significantly different in the two experimental stages [416 vs. $420 \mathrm{msec}$ in the TRS and TSS; $F(1,11)=0.55, p=.475]$ and decreased with increasing ISI [441, 412, and $400 \mathrm{msec}$ for ISI 0,53 , and 80, respectively; $F(2,22)=85.32, p<.001]$. Mean responses were longer in congruent than in incongruent trials [425 vs. $410 \mathrm{msec}$, respectively; $F(1,11)=18.21, p=.001]$.

The stage $X$ congruency interaction was significant $[F(1,11)=6.57, p=.026]$, and contrast analysis showed that the priming effect was negative in both the TSS $[-20 \mathrm{msec} ; F(1,11)=19.32, p=.001]$ and the TRS $[-9$ msec; $F(1,11)=8.27, p=.015]$. The interaction reflects the greater magnitude of inverse priming in the TSS in comparison with the TRS.

Similarly, we found a significant congruency $\times$ ISI interaction $[F(2,22)=5.68, p=.010]$. Contrast analysis indicated that the priming effect was inverse but was significant only for ISI 53 [423 vs. $401 \mathrm{msec}$ for congruent and incongruent trials, respectively; $F(1,11)=29.40, p=$ $.000]$ and ISI 80 [410 vs. $391 \mathrm{msec} ; F(1,11)=15.98, p=$ $.002]$, not for ISI 0 [444 vs. $439 \mathrm{msec} ; F(1,11)=0.79$, $p=.395]$.

Again, the three-way interaction (stage $\times$ ISI $\times$ congruency) was not significant $[F(2,22)=0.234, p=.794]$.

Error rates. Percentages of incorrect responses were almost the same in both TI blocks [8\% vs. $7 \%$ in the TRS and TSS, respectively; $F(1,11)=1.81, p=.206]$ and increased with ISI $[6 \%, 7 \%$, and $10 \%$ for ISI 0,53 , and 80 ,

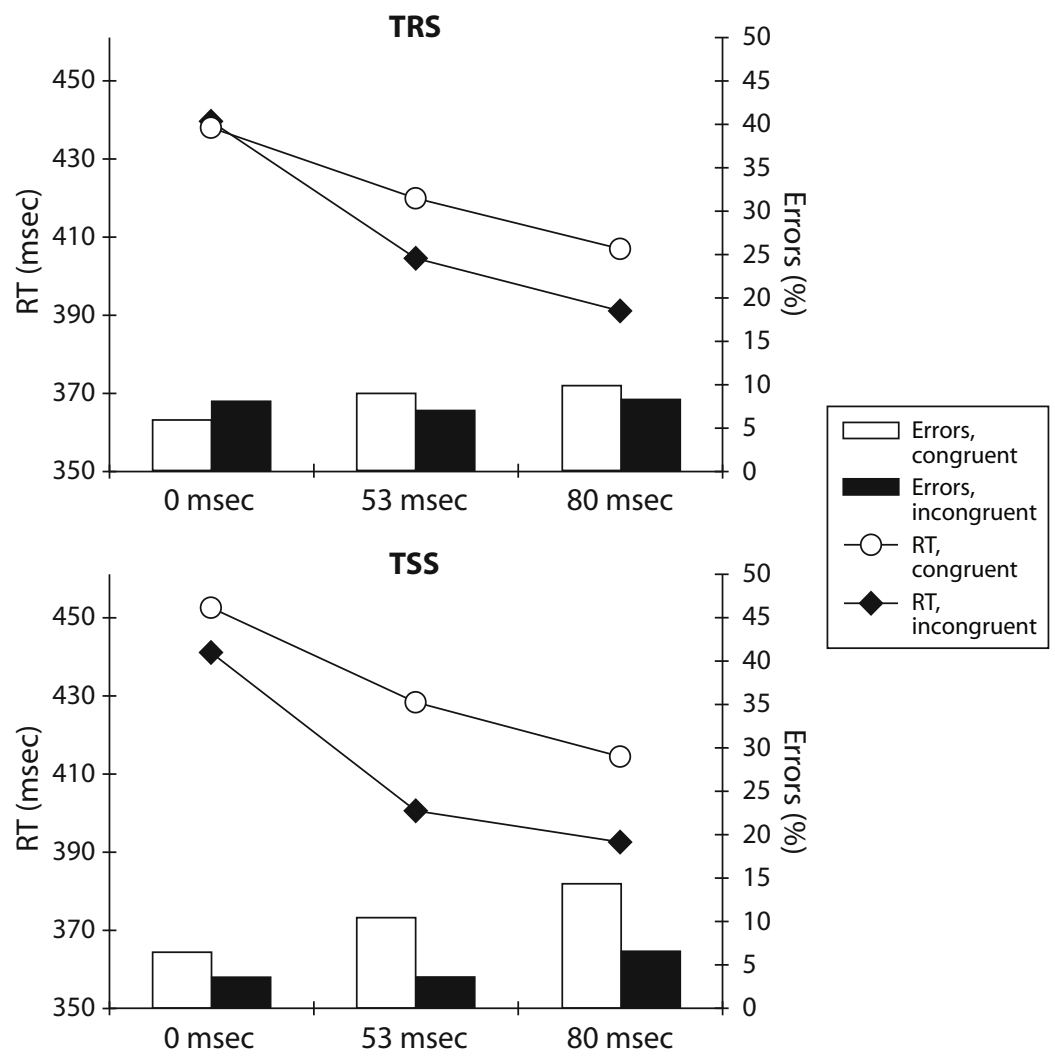

Figure 5. Mean reaction times (RTs) and error rates from Experiment 5, as a function of interstimulus interval. (Top) Training stage. (Bottom) Test stage. For RTs, congruent trials are denoted by white circles and incongruent trials by black diamonds. For errors, white bars are results for congruent trials and black for incongruent trials. 
respectively; $F(2,22)=6.38, p=.007]$. The mean priming effect was not significant $[F(1,11)=3.81, p=.077]$.

We found a significant interaction between stage and congruency $[F(1,11)=12.90, p=.004]$. This was due to inverse priming in the TSS $[10 \%$ vs. $4 \%$ for congruent and incongruent trials, respectively; $F(1,11)=9.15$, $p=.011]$ versus a lack of priming in the TRS [8\% vs. $8 \% ; F(1,11)=0.20, p=.660]$, as revealed by separate contrast analyses.

The interactions ISI $\times$ congruency $[F(2,22)=2.89$, $p=.077]$ and stage $\times$ ISI $\times$ congruency $[F(2,22)=$ $0.21, p=.813$ ] were not significant.

\section{Discussion}

The results of Experiment 5 showed that the priming effect became increasingly negative with the elongation of the time interval between mask and target. This increase in the priming effect started from 0 in the TRS and from $-11 \mathrm{msec}$ in the TSS.

These findings are consistent with our hypothesis, according to which more complex masks take longer to be processed. With short mask-target intervals, the visual system is not able to find relevant elements in the mask, so the interaction between prime and mask is weak. Once the ISI becomes longer, the interaction comes into play. After training, however, recognition is more efficient, and the interaction becomes observable even for short ISIs.

\section{GENERAL DISCUSSION}

In the present study, we have demonstrated that inverse priming increases with training: The effect was initially straight or absent, but gradually it became more and more inverse. We showed that these changes over time critically depend on whether the mask precedes the target and on the complexity of the masking pattern. We argue that these findings are consistent with the hypothesis that (1) mask content interacts with information provided by the prime, and thereby modulates the priming effect, and (2) during the task, participants improve their ability to recognize the mask content.

The present research follows directly from Klapp and Hinkley's (2002) earlier observations. We replicated their finding that inverse priming appears after some practice. In their experiments, however, inverse priming was only observed on the second and third days of testing, whereas we found the effect as early as in the second part of the (first) session. Therefore, one might suggest that the effects demonstrated by Klapp and Hinkley and by us are the outcomes of two different mechanisms. We believe, however, that these were two demonstrations of the same phenomenon. Indeed, consistent with Klapp and Hinkley's results, we had no general priming effect in the majority of our conditions. It is quite likely that Klapp and Hinkley would have noticed a similar effect of practice in their first session if they had split it into two parts. It is also possible that we would have found stable priming in second and third sessions.

Moreover, Klapp and Hinkley's (2002) failure to obtain negative priming in the first session has not been common in the literature; typically, negative priming has appeared in the first session. The practice effect showed by Klapp and Hinkley may have resulted from mask complexity. Indeed, we showed that the magnitude of the priming effect depends on mask complexity, since it is generally smaller when the masking pattern is more complex, in the sense that extracting task-relevant objects from the mask is more difficult. The masks used by Klapp and Hinkley could be considered complex: The masks were composed of letters (WXXW and XWwX), but one could speculate that they nonetheless contained the searched-for features (arrows). Both of the letters used (especially x) might be perceived as a compound of two opposing arrows. According to our hypothesis, the sessions performed on three consecutive days in Klapp and Hinkley's study enabled the visual system to improve searching for arrow elements in the masks, so that inverse priming became stronger.

How do our results relate to the SI and OU hypotheses presented in the introduction?

The OU (Lleras \& Enns, 2004) and AM (Verleger et al., 2004) hypotheses suggest that the mask content induces inverse priming. At first glance, failure to find inverse priming with an arrow mask at the beginning of an experimental session argues against the two hypotheses, since the arrow mask should interact with the preceding prime. However, this would be possible only if the relevant or arrow-like elements were clearly recognizable. If not, such a mask would work like a mask composed of task-irrelevant elements. Indeed, in our Experiment 4 we showed that an initial lack of inverse priming did not occur with a simple mask in which the arrow-like elements were clearly recognizable. If a mask has a more complex pattern, training is necessary to improve participants' ability to recognize the mask content. Thus, our results are in perfect agreement with the two hypotheses. Further systematic study should explain whether the learning effect applies only to arrows, as Verleger et al. (2004) argued, or to relevant elements in general.

In the SI hypothesis, the role of the mask is restricted to reducing prime visibility and removing prime-related input (Eimer et al., 2002; Klapp \& Hinkley, 2002; Schlaghecken \& Eimer, 2002). Our findings, on the contrary, strongly suggest that the mask plays an active role and that it can modulate the priming effect. Thus, this hypothesis in its original formulation is difficult to maintain. It could be saved, however, if one assumes that at least one more mechanism is involved in which the prime-mask interaction plays an important role. Such a complement of the SI hypothesis was recently suggested by Bowman, Schlaghecken, and Eimer (2006). They admitted that selfinhibition cannot be an exclusive mechanism responsible for inverse priming and that prime-mask interaction (like that suggested by the OU hypothesis) is plausible. In other words, self-inhibition operates with all kinds of masks, whereas the other mechanism comes into play once the mask possesses relevant features. Our findings are consistent with such a weakened version of the SI hypothesis. Since we used only relevant masks, all changes of the priming effect could indeed be assigned to the primemask interaction. 
An alternative to the perceptual-learning account is that inverse priming exists from the very beginning, but its time course changes systematically with practice. Lingnau and Vorberg (2005) showed recently that the magnitude and sign of the priming effect changed markedly with the mask-target interval. The findings presented in the present report are consistent with such an account. Although this is an empirical rather than a theoretical hypothesis, it seems worthy of further investigation.

To sum up, we have clearly demonstrated that a mask is not a neutral stimulus whose role is restricted merely to reducing prime visibility, but rather that it can modulate, depending on content, prime-induced motor preparation. In the course of an experiment, participants become more and more sensitized to the task-relevant/arrow-like features and are able to recognize them more efficiently in the masking pattern. This in turn leads to an increasingly strong interaction between prime and mask.

\section{AUTHOR NOTE}

Correspondence relating to this article may be sent to A. PrzekorackaKrawczyk, Quantum Electronics Laboratory, Faculty of Physics, Adam Mickiewicz University, Umultowska 85, Poznań, Poland (e-mail: aniaprzek@poczta.onet.pl).

\section{REFERENCES}

Ansorge, U., Klotz, W., \& Neumann, O. (1998). Manual and verbal responses to completely masked (unreportable) stimuli: Exploring some conditions for the metacontrast dissociation. Perception, 27, 1177-1189.

Bowman, H., Schlaghecken, F., \& Eimer, M. (2006). A neural network model of inhibitory processes in subliminal priming. Visual Cognition, 13, 401-480.

Di Lollo, V., Bischof, W. F., \& Dixon, P. (1993). Stimulus-onset asynchrony is not necessary for motion perception or metacontrast masking. Psychological Science, 4, 260-263.

EIMER, M. (1999). Facilitatory and inhibitory effects of masked prime stimuli on motor activation and behavioural performance. Acta Psychologica, 101, 293-313.

Eimer, M., \& Schlaghecken, F. (1998). Effects of masked stimuli on motor activation: Behavioral and electrophysiological evidence. Journal of Experimental Psychology: Human Perception \& Performance, 24, 1737-1747.

Eimer, M., \& Schlaghecken, F. (2001). Response facilitation and inhibition in manual, vocal, and oculomotor performance: Evidence for a modality-unspecific mechanism. Journal of Motor Behavior, 33, 16-26.

Eimer, M., Schubö, A., \& Schlaghecken, F. (2002). Locus of inhibition in the masked priming of response alternatives. Journal of Motor Behavior, 34, 3-10.

ERDELYI, M. H. (2004). Subliminal perception and its cognates: Theory, indeterminacy, and time. Consciousness \& Cognition, 13, 73-91.

JAŚKOWSKI, P. (2006). Inverse priming: Which perceptual features of the mask do matter? Unpublished manuscript.

JAŚKOWSKi, P., \& PrZEKORACKA-KraWCZYK, A. (2005). On the role of mask structure in subliminal priming. Acta Neurobiologiae Experimentalis, 65, 409-417.

JaŚKowski, P., Skalska, B., \& Verleger, R. (2003). How the self controls its "automatic pilot" when processing subliminal information. Journal of Cognitive Neuroscience, 15, 911-920.

JAŚKOWSKI, P., \& ŚLÓSAREK, M. (2007). How important is prime gestalt for subliminal priming? Consciousness \& Cognition, 16, 485-497.

KLAPP, S. T., \& HinkLEY, L. B. (2002). The negative compatibility effect: Unconscious inhibition influences reaction time and response selection. Journal of Experimental Psychology: General, 131, 255-269.

LingnaU, A., \& Vorberg, D. (2005). The time course of response inhibition in masked priming. Perception \& Psychophysics, 67, 545-557.

Lleras, A., \& EnNS, J. T. (2004). Negative compatibility or object updating? A cautionary tale of mask-dependent priming. Journal of Experimental Psychology: General, 133, 475-493.

Praamstra, P., Boutsen, L., \& Humphreys, G. W. (2005). Frontoparietal control of spatial attention and motor intention in human EEG. Journal of Neurophysiology, 94, 764-774.

SchlaghecKen, F., \& Eimer, M. (1997). The influence of subliminally presented primes on response preparation. Sprache \& Kognition, 16, $166-175$.

Schlaghecken, F., \& EIMER, M. (2000). A central-peripheral asymmetry in masked priming. Perception \& Psychophysics, 62, 1367-1382.

Schlaghecken, F., \& Eimer, M. (2001). Partial response activation to masked primes is not dependent on response readiness. Perceptual \& Motor Skills, 92, 208-222.

Schlaghecken, F., \& Eimer, M. (2002). Motor activation with and without inhibition: Evidence for a threshold mechanism in motor control. Perception \& Psychophysics, 64, 148-162.

VERLEGER, R., EWERs, T., \& JAŚKOWSKI, P. (2006). On the special role of arrow-masked arrows as flankers: Inverse priming depends on types of stimuli and of masks. Unpublished manuscript.

VERLEGER, R., Görgen, S., \& JAŚKOWSKI, P. (2005). An ERP indicator of processing relevant gestalts in masked priming. Psychophysiology, 42, 677-690.

Verleger, R., JAŚKowski, P., Aydemir, A., VAn der Lubbe, R. H. J., \& GROEN, M. (2004). Qualitative differences between conscious and nonconscious processing? On inverse priming induced by masked arrows. Journal of Experimental Psychology: General, 133, 494-515.

Verleger, R., Vollmer, C., Wauschiuhn, B., VAN Der Lubbe, R., \& WAScher, E. (2000). Dimensional overlap between arrows as cueing stimuli and responses? Evidence from contra-ipsilateral differences in EEG potentials. Cognitive Brain Research, 10, 99-109.

\section{NOTES}

1. A contrast analysis showed that the priming effect obtained in Experiment 2 was significantly lower than the mean effect obtained across Experiments 1 and $3[F(1,30)=4.16, p=.050]$.

2. Participants complain very often that the PD task with perfect masking is very boring and frustrating. Therefore, the results may often be unreliable, because participants seeing nothing may have lost their motivation to attend to the stimuli. Therefore, we added two prime durations longer than the one used in the target identification block, since we reasoned that this should help improve their performance by increasing their motivation, because they could feel that they were doing well on a significant number of trials.

(Manuscript received October 11, 2005; revision accepted for publication September 11, 2006.) 